\title{
COMPLEX STRUCTURAL REARRANGEMENT OF CHROMOSOMES 7, 10, 14 AND 21
}

\author{
Ma. Luisa de Asis, * Fumiko Saito, and Akira Tonomura \\ Department of Cytogenetics, Medical Research Institute, Tokyo Medical \\ and Dental University, Yushima I-Chome, Bunkyo-ku, Tokyo 113, Japan
}

\begin{abstract}
Summary This paper reports a rare case of complex structural rearrangement involving four chromosomes. The patient, a five-year old girl, exhibits mild physical deformities, such as hypertelorism, blepharophimosis, brachydactyly and clinodactyly of both the second and fifth fingers. Using various banding techniques, the complicated multiple rearrangement could be interpreted as a unique combination of non-reciprocal translocation and insertion involving chromosomes 7, 10, 14 and 21.
\end{abstract}

\section{INTRODUCTION}

Complex chromosome rearrangements (CCR) involving three or more chromosomes are not common. According to the manner of transmission, all CCR can be grouped into two: CCR with familial occurrence and CCR arising de novo (Bass and Sparker, 1979; Muneer et al., 1981; Joseph and Thomas, 1982; Kleczkowska et al., 1982). About 21 cases of the familial type and 19 cases arising de novo have been summarized by Kleczkowska et al. (1982). In some of these reported cases, the subjects carrying the translocations were phenotypically normal (Buchanan et al., 1978).

The recent developments in differential staining techniques have resulted to a more precise delineation of complex rearrangement. However, in these cases involving multiple breakpoints, there is always a risk of minute deletion of euchromatic segments which are often beyond the high resolution of present banding techniques (Muneer et al., 1981).

In this paper, we report an additional case of complex chromosome rearrangement involving four chromosomes. Clinical findings, dermatoglyphic features and cytogenetic studies are presented.

Received August 15, 1984

* Present address: Department of Physics, De La Salle University, Manila, Philippines. 


\section{CASE REPORT}

The patient, a five-year old girl, was referred to the laboratory for chromosome evaluation. She was $2,320 \mathrm{~g}$ at birth and was the second child of a normal couple. Her mother was 28 years old and her father was 29 years old at the time of her birth. The first child was a normal female.

On examination, the following clinical features were noted: Hypertelorism, blepharophimosis, small nose (Fig. 1), brachydactyly (with mid-phalangial hypoplasia from the second to the fifth fingers), clinodactyly of both the second and fifth fingers and only one crease on the right fifth finger.

Dermatoglyphic study revealed that she has all whorl patterns on the fingertips. The same was true for both parents. Dermatoglyphic formulas of the patient and her parents are shown in Table 1.

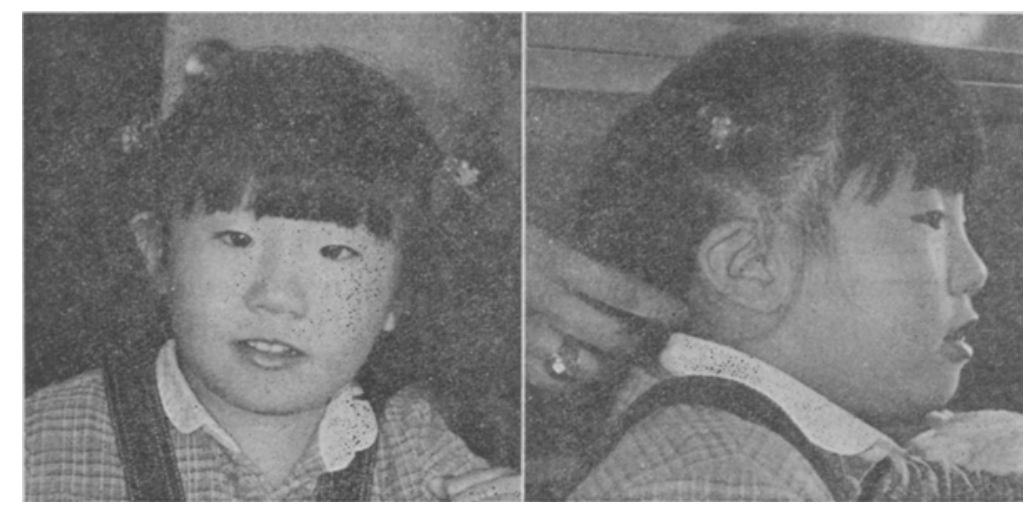

Fig. 1. The patient at age five years, who appears normal except for some mild deformities.

Table 1. Dermatoglyphic features of the patient and her parents.

\begin{tabular}{|c|c|c|c|c|c|c|c|}
\hline \multirow{2}{*}{\multicolumn{2}{|c|}{ Subjects }} & \multicolumn{5}{|c|}{ Digits } & \multirow{3}{*}{$\frac{\text { Palmer formula }}{\text { 9.7.4.-t-O.O/O.O.L.O }}$} \\
\hline & & \multirow{2}{*}{$\frac{I}{W}$} & \multirow{2}{*}{$\frac{\text { II }}{W}$} & \multirow{2}{*}{$\frac{\text { III }}{W}$} & \multirow{2}{*}{$\frac{\text { IV }}{W}$} & \multirow{2}{*}{$\frac{\mathrm{V}}{\mathrm{W}}$} & \\
\hline Patient & Right & & & & & & \\
\hline & Left & W & W & W & W & W & 11.9.7.4.-t-O.O/O.O.L.O \\
\hline \multirow[t]{2}{*}{ Father } & Right & W & W & W & W & W & 9.7.5.4.-t-O.O/O.O.L.O \\
\hline & Left & W & W & W & $\mathrm{W}$ & W & 0.5.5.3.-t-0.0/0.0.0.0 \\
\hline \multirow[t]{2}{*}{ Mother } & Right & W & W & w & $\mathrm{W}$ & W & 11.9.7.11.-t-O.Au/O.O.L.O \\
\hline & Left & $\mathrm{W}$ & $\mathrm{W}$ & W & W & $\mathrm{W}$ & 11.9.7.3.-t-O.Au/O.O.L.O \\
\hline
\end{tabular}




\section{CYTOGENETIC STUDIES}

Routine chromosome preparations from cultured peripheral blood lymphocytes revealed 46 chromosomes with morphological abnormalities. All metaphase cells studied by conventional staining revealed that one of the $\mathrm{C}$ group chromosomes had an isochromatid gap, seven D group chromosomes were present but one was completely telocentric, one extra $\mathrm{E}$ group chromosome was identified its arm ratio resembled that of 17 and one of the $\mathrm{G}$ group chromosomes was missing.

Trypsin Giemsa banding was carried out according to a slight modification of the standard method (Seabright, 1971) and interpretation of the G bands indicated the occurrence of a complex rearrangement following seven breaks. These breaks produced four abnormal chromosomes 7,10,14 and 21. Breakage has occurred at or near the centromere of chromosome 7 and produced the abnormal 7 with dele-

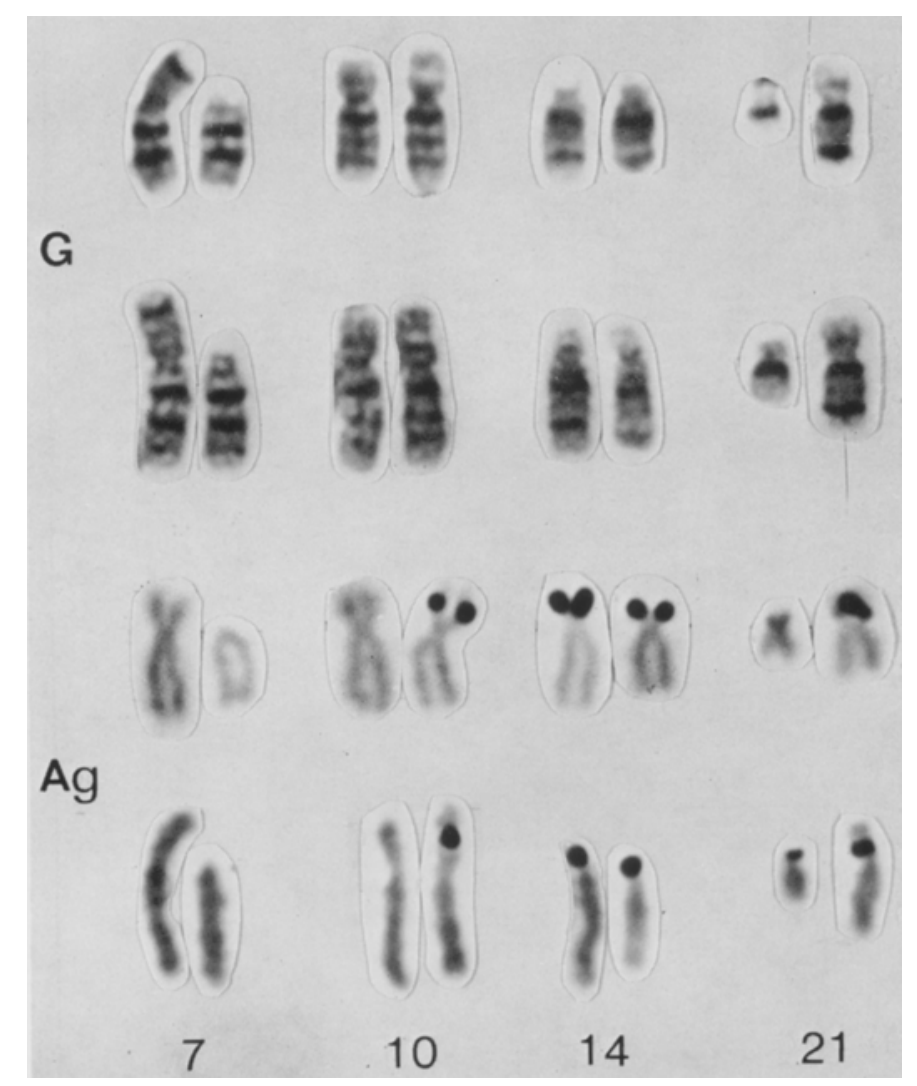

Fig. 2. G-banded (G) and Ag-stained (Ag) partial karyotypes of the patient showing the normal and abnormal chromosomes $7,10,14$ and 21 . 


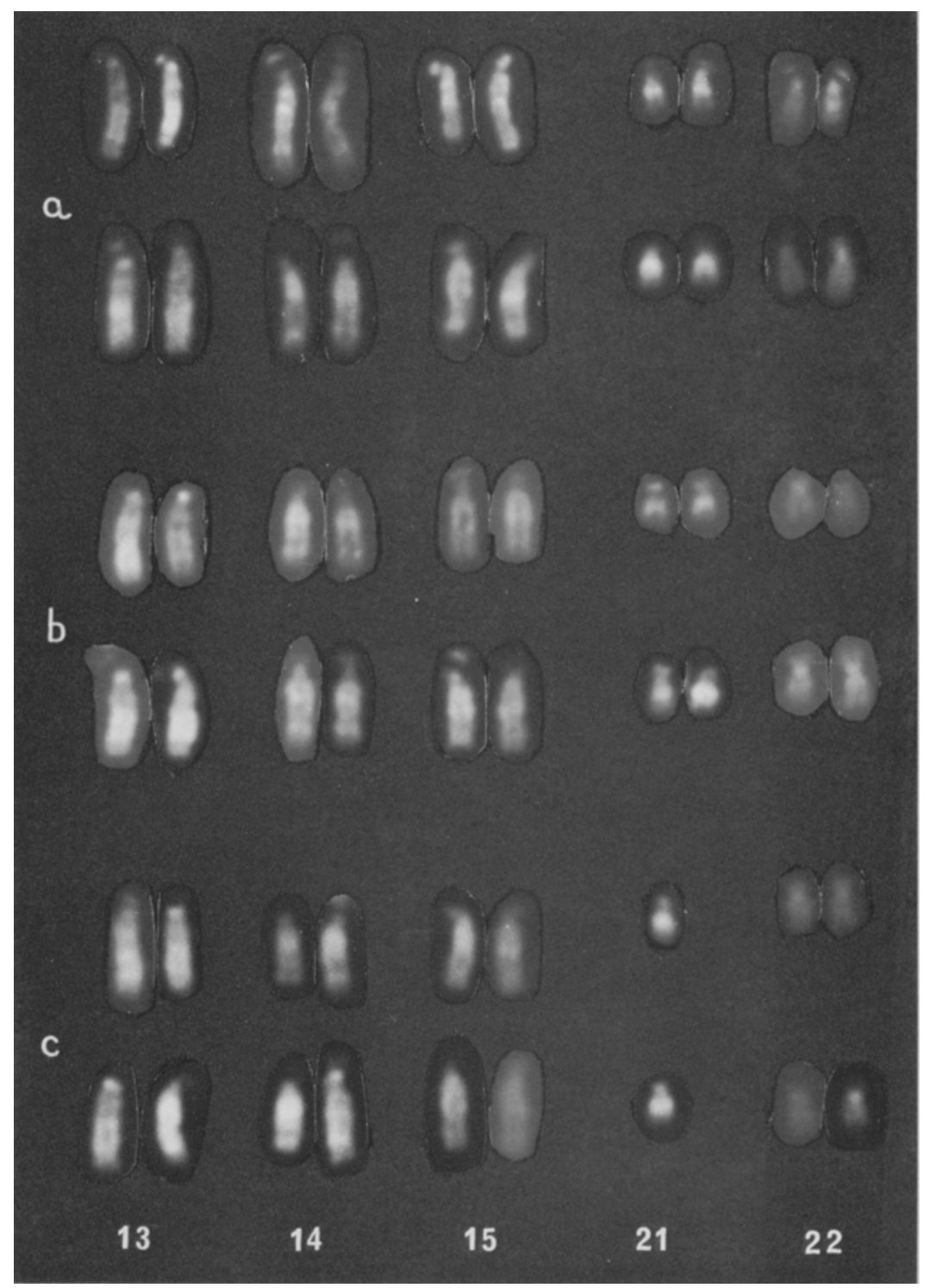

Fig. 3. Q-banded partial karyotypes of the father (a), mother (b) and the patient (c) showing the $\mathrm{D}$ and $\mathrm{G}$ group chromosomes.

tion of short arm. The abnormal 10 appeared to have a secondary constriction lying between two bands pl2 and p14. The abnormal 14 showed a break at band $\mathrm{q} 32$ and deletion of the terminal region of the long arm. The abnormal 21 seemed to contain the translocated segments of the other abnormal chromosomes. It can 
be described to have a proximal band in its short arm and two dark bands at the proximal and distal parts of the long arm. With silver staining method, in addition to the acrocentric chromosomes, the region of the secondary constriction in the abnormal 10 and the short arm of the abnormal 21 stained positively (Fig. 2).

To ascertain the origin of the complex translocation, karyotype analyses were performed on both parents. Results revealed normal karyotypes.

Interpretation derived from $Q$ banding patterns supported the results of $G$ banding. On the basis of similarity of satellites of acrocentric chromosomes the intact chromosome 21 seemed to be of maternal origin (Fig. 3). The abnormal 21 to which the broken segments from other chromosomes have translocated is believed to be of paternal origin. The terminally deleted portions of the abnormal 14 and 21 are difficult to ascertain even with the use of these bandings.

Considering the results of all the banding techniques, presumptive breakpoints on the chromosomes involved in the CCR are given in Fig. 4. The complex structural rearrangement of the chromosomes was interpretated as follows: a part of the broken short arm of chromosome 7 ( $7 \mathrm{pcen} \rightarrow 7 \mathrm{p} 13)$ was inserted between the proximal part of the stalk of chromosome 21 at p12 and the translocated terminal region of chromosome 14(?); the remaining portion of the short arm of chromosome 7 $(7 \mathrm{p} 13 \rightarrow 7 \mathrm{pter})$ translocated to terminally broken long arm of chromosome 21 , broken at or near band q22; the silver positive region in the short arm of chromosome 10 is believed to be a part of the stalk of chromosome 21 that has inserted between the broken segment of chromosome 10 at or near p12; the precise location of the terminally deleted portions of chromosomes 14 and 21 could not be determined (note broken lines in Fig. 4).

\section{DISCUSSION}

A seven-break non-reciprocal translocation and insertion is proposed to explain the morphology of the abnormal chromosomes as shown by their $\mathrm{G}$ and $\mathrm{Q}$ banding patterns. Figure 4 shows the presumptive breakpoints on the chromosomes involved in the rearrangement. Of these, fates of the terminal fragments of chromosomes 14 and 21 cannot be determined with certainty. 'Quite a number of studies have pointed out that chromosome 14 is the chromosome most often involved with chromosome 21 (Schwinger et al., 1975). Thus we may suppose that it is the terminal region of $14 \mathrm{q}$ which joined with the short arm of abnormal 21 and that the terminal region of $21 \mathrm{q}$ translocated to or near the centromere of abnormal 7 .

Assuming that there is no apparent loss of chromosome material, this CCR is mildly expressed phenotypically in the child. This may suggest that clinical abnormalities are produced by position effects. As has already been mentioned by Fitzgerald (1974), displacement of genes from their normal positioning along the chromosomes brought about by the translocation could disturb the normal functioning of some of the genes. However, since the possibility of minute deletion could not 


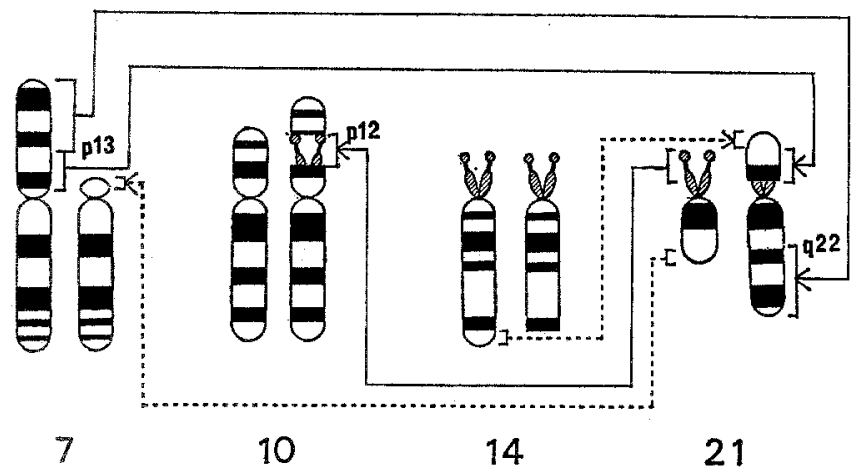

Fig. 4. Idiogram showing the presumptive breakpoints on the chromosomes involved in the complex rearrangement.

totally ruled out, the loss of chromosome material could also account for the dysmorphic features.

It is also quite interesting to note that a familial case involving the same chromosomes 7, 10, 14 and 21 has been previously reported by Bass and Sparkers (1979). They described double translocation heterozygosity in three generations. One was a reciprocal rearrangement between chromosomes 7 and 10 and the other was a Robertsonian translocation involving chromosomes 14 and 21. The mechanism and breakpoints involved are, however, different from the present de novo case. But this study agrees with the previous findings on balanced and unbalanced complex translocations involving three or more chromosomes that some chromosomes are more frequently involved in these structural rearrangement (Kleczkowska et al., 1981).

The CCR seems to occur either in a germinal cell or in early embryonic life. Based on the size and staining intensity of satellite region of the intact chromosome 21 , the abnormal 21 is believed to be of paternal origin in the present de novo case. However, the precise delineation of where and when the CCR may have risen requires a considerable study.

\section{REFERENCES}

Bass, H.N. and Sparker, R.S. 1979. Two balanced translocations in three generations of a pedigree: $t(7 ; 10)(\mathrm{q} 11 ; \mathrm{q} 22)$ and $\mathrm{t}(14 ; 21)(14 \mathrm{qter} \rightarrow \mathrm{cen} \rightarrow 21 \mathrm{qter}) . \quad J$. Med. Genet. 16: 215-218.

Buchanan, P.D., Rao, K.W., Doerr, C.L., and Aylsworth, A.S. 1978. A complex translocation involving chromosomes 3,11 and 14 which an interstitial deletion, del(14)(q13q22) in a child with congenital glaucoma and cleft lip and palate. Birth Defects 14: 317-322.

Fitzgerald, M.G. 1974. Complex five-break rearrangement. Clin. Genet. 5: 62-67.

Joseph, A. and Thomas, I.M. 1982. A complex rearrangement involving three autosomes in a phenotypically normal male presenting sterility. J. Med. Genet. 19: 375-377. 
Kleczkowska, A., Fryns, J.P., and Van Den Berghe, H. 1982. Complex chromosomal rearrangements (CCR) and their genetic consequence. J. Génét. Hum. 30: 199-214.

Muneer, R.S., Donaldson, D.L., and Rennert, O.W. 1981. Complex balanced translocation of chromosomes 2, 3, and 13. Hum. Genet. 59: 182-184.

Seabright, M. 1971. A rapid banding technique for human chromosomes. Lancet II: 971-972.

Schwinger, E., Mikkelsen, M., and Niesen, M. 1975. Familial balanced $(7 ; 11 ; 21)$ translocation and Down's syndrome in two siblings. Clin. Genet. 7: 304-307.

Vol. 29, No. 4, 1984 